\title{
Bericht über die 5I. Internationale Mathematik-Olympiade
}

Hans-Dietrich Gronau

Die 5I. Internationale Mathematik-Olympiade (IMO) fand vom 2. bis zum 14. Juli in Astana, Kasachstan statt. 97 Länder und 517 Schülerinnen und Schüler nahmen an dieser Olympiade teil, was sie im Hinblick auf die Teilnehmerzahl zur viertgrößten IMO macht.

Die deutsche Mannschaft bestand aus einer Schülerin und fünf Schülern (Lisa Sauermann, Simon Buchholz, Fabian Grundlach, Jens Reinhold, Michael Schubert und Florian Schweiger), dem Berichterstatter als Delegationsleiter, Dr. Christian Reiher als stellvertretendem Delegationsleiter und Dr. Peter Wagner als Beobachter.

\section{Auswahl und Vorbereitung der deutschen Mannschaft}

Die Auswahl und Vorbereitung der deutschen Mannschaft erfolgte nach dem Verfahren der Vorjahre. Insgesamt 136 Schülerinnen und Schüler qualifizierten sich durch die erfolgreiche Teilnahme an der 2. Runde des Bundeswettbewerbs Mathematik oder an der Deutschland-Olympiade, der 4. Stufe der MathematikOlympiaden, für die zwei Auswahlklausuren, die Anfang Dezember 2009 geschrieben wurden. Die 16 erfolgreichsten der 107 Klausurteilnehmer bildeten den Kandidatenkreis für die deutsche Mannschaft. Die Kandidaten nahmen an einwöchigen Seminaren in Rostock, drei Wochenenden in Bad Homburg und der traditionellen Abschlusswoche am Mathematischen Forschungsinstitut in Oberwolfach teil, wobei die beiden längeren Kurse unter der Leitung des Berichterstatters standen. Während dieser Zeit wurden insgesamt sieben Klausuren geschrieben, und die sechs besten Kandidaten qualifizierten sich für die IMO-Mannschaft.

Zusätzlich hat die Mannschaft wie im vergangenen Jahr ein selbst organisiertes Intensivtraining - diesmal in Bielefeld - durchgeführt, das sich als sehr konstruktiv erwiesen hat. Große Anerkennung an das Team!

Schließlich gab es ein Wochenendseminar an der JacobsUniversity Bremen.

Das seit 2007 von der Deutsche Telekom Stiftung unterstütze Programm ,Jugend trainiert Mathematik“ (JuMa) zeigt, dass es sehr gut greift, denn fünf Mitglieder des diesjährigen Teams haben das Programm erfolgreich durchlaufen. (Nähere Informationen unter http://www. bundeswettbewerb-mathematik.de/juma/)

Die gesamte organisatorische Vorbereitung und Durchführung der Klausuren, der Seminare, der Reise etc. wurden wieder vom IMO-Organisationsbüro unter Leitung von Herrn H.-H. Langmann in gewohnt perfekter Weise gestaltet. Ihm sei herzlich gedankt!

\section{Der Ablauf der 5 I. IMO}

Die Schüler reisten mit dem stellvertretenden Delegationsleiter am 5. Juli nach Astana, der neuen Hauptstadt Kasachstans. Die Eröffnungsveranstaltung fand am 6. Juli im Unabhängigkeitspalast in Astana statt. Neben der Begrüßung durch den Minister für Bildung und Wissenschaft und kulturellen Darbietungen bildete die Parade aller teilnehmenden Länder den Höhepunkt. Anschließend mussten die Teams noch bis in das ca. $250 \mathrm{~km}$ entfernte „Republican Educational and Practical Center Baldauren“ weiterfahren, wo in den darauffolgnden Tagen unter guten Bedingungen die Klausuren geschrieben wurden. Die beiden folgenden Tage waren der Durchsicht und Bewertung der Klausuren gewidmet.

Die Preisverleihung fand am I3. Juli wieder im Unabhängigkeitspalast statt, abgeschlossen von einem zweistündigen „Farewell Dinner“. Die Rückreise erfolgte am frühen Morgen des 14. Juli.

Jedes Team wird bei der IMO üblicherweise von einem Guide betreut. Unsere Mannschaft hatte in diesem Jahr wiederum großes Glück, denn unsere Betreuerin war sehr engagiert. Ihr sei herzlich dafür gedankt!

\section{Der Wettbewerb}

Leider wurde die 5I. IMO wurde durch einen Betrug überschattet, der zur Disqualifikation der nordkoreanischen Mannschaft führte. In der Geschichte der IMO kam es bislang nur zweimal zur Disqualifikation eines ganzen Teams, 1991 und in diesem Jahr (im Jahr 1991 war es ebenfalls das Team aus Nordkorea, das disqualifiziert wurde).

Bei der IMO wurden 36,I \% der möglichen Punkte erreicht. Sie war somit fast genauso schwer wie in den bei- 


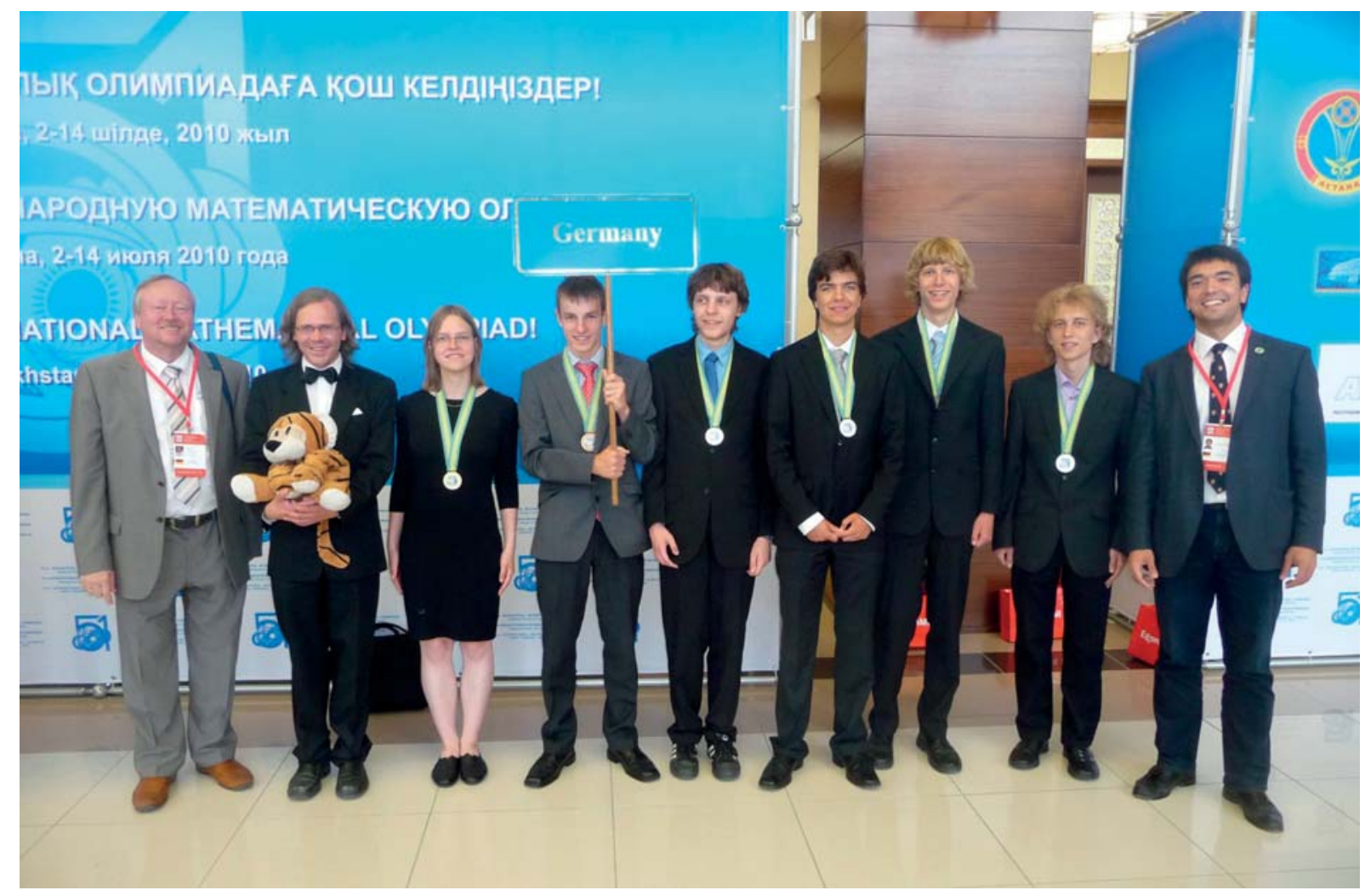

Die deutsche Mannschaft: Prof. Dr. Hans-Dietrich Gronau, Dr. Christian Reiher, Lisa Sauermann, Michael Schubert, Fabian Gundlach, Jens Reinhold, Simon Buchholz, Florian Schweiger, Dr. Peter Wagner (v. I. n. r.)

den Vorjahren. Allerdings fiel die Aufgabe 5 etwas aus dem Rahmen, da sie sich für die stärkeren Teams überraschend als schwerste Aufgabe herausstellte, während Dänemark, das in der inoffiziellen Gesamtwertung auf Platz 45 liegt, mit China und den USA gewann.

Die volle Punktzahl erreichte nur ein Schüler aus China. Es folgen ein Schüler mit 39 Punkten aus den USA, einer mit 37 Punkten aus Serbien und 3 Teilnehmende mit 36 Punkten. Darunter ist auch Lisa Sauermann, die damit schon ihre dritte Goldmedaille gewann. Insgesamt war diese IMO auch für die Allerbesten sehr schwer. Die Siegermannschaft aus China erreichte 78,2\% der möglichen Punkte. In den vergangenen 30 Jahren hatte nur dreimal die Siegermannschaft weniger Punkte.

Wir freuen uns sehr, dass mit Lisa Sauermann wieder jemand in den exklusiven „Club der IMO-Teilnehmer mit mindestens 3 Goldmedaillen“" (siehe die Webseite www. Mathematik-Olympiaden.de oder www.imo-official.org/ hall.aspx) aufgenommen werden konnte. Unter diesen 33 Erfolgreichsten gibt es nunmehr zwei Mädchen. Von den insgesamt 13428 Teilnehmenden aller bisherigen IMOs gelang es bislang nur zweien, vier Goldmedaillen zu gewinnen: Christian Reiher (Deutschland) und Reid Barton (USA).
In diesem Jahr erfolgte eine geringfügige Modifikation des seit Jahren festgeschriebenen Reglements, das vorsieht, dass nicht mehr als die Hälfte der Teilnehmer einen Preis erhält. Da bei einer strikten Anwendung der Regeln lediglich 43,7\% der Teilnehmenden einen Preis erhalten hätten, erlaubte der Chairman der Jury eine Abstimmung, bei der mit großer Mehrheit dafür gestimmt wurde, die Punktgrenze für Bronze um einen Punkt auf I5 Punkte herabzusetzen.

Detaillierte Informationen zu den Ergebnissen der Länder und denen der einzelnen Teilnehmenden sind auf der Seite www.imo-official.org zu finden.

\section{Ausblick}

Traditionell hätte schon vor einem Jahr das Austragungsland für die IMO 2013 bestimmt werden sollen. Doch weder 2009 noch in diesem Jahr fand sich ein ernsthafter Bewerber. Kommendes Jahr wird die IMO in Amsterdam, Niederlande stattfinden, 2012 in Argentinien.

Prof. Dr. Hans-Dietrich Gronau, Institut für Mathematik, Universität Rostock, I805I Rostock. gronau@uni-rostock.de 
I. Tag

I. Man bestimme alle Funktionen $f: \mathbb{R} \rightarrow \mathbb{R}$, so dass die Gleichung

$$
f(\lfloor x\rfloor y)=f(x)\lfloor f(y)\rfloor
$$

für alle $x, y \in \mathbb{R}$ gilt.

(Frankreich)

2. Das Dreieck $A B C$ habe den Inkreismittelpunkt / und den Umkreis $\Gamma$. Die Gerade $A /$ schneide $\Gamma$ ein zweites Mal im Punkt $D$. Ferner seien $E$ ein Punkt auf dem Bogen $B D C$ und $F$ ein Punkt auf der Seite $\overline{B C}$ mit

$$
\Varangle B A F=\Varangle C A E<\frac{1}{2} \Varangle B A C .
$$

Schließlich sei $G$ der Mittelpunkt der Strecke $\overline{I F}$. Man beweise, dass sich die Geraden $D G$ und $E$ l auf $\Gamma$ schneiden.

(Hongkong)

3. Es sei $\mathbb{N}^{+}$die Menge der positiven ganzen Zahlen. Man bestimme alle Funktionen $g: \mathbb{N}^{+} \rightarrow \mathbb{N}^{+}$, so dass die Zahl

$$
(g(m)+n)(m+g(n))
$$

für alle $m, n \in \mathbb{N}^{+}$eine Quadratzahl ist.
2. Tag

4. Im Inneren des Dreiecks $A B C$ liege der Punkt $P$. Die Geraden $A P, B P$ und $C P$ schneiden den Umkreis $\Gamma$ von $A B C$ jeweils ein zweites $M a l$ in den Punkten $K, L$ bzw. $M$. Die Tangente an $\Gamma$ durch $C$ schneide die Gerade $A B$ in $S$. Es gelte $|\overline{S C}|=|\overline{S P}|$.

Man beweise $|\overline{M K}|=|\overline{M L}|$.

(Polen)

5. In jedem von sechs Behältern $B_{1}, B_{2}, B_{3}, B_{4}, B_{5}$ und $B_{6}$ befindet sich zu Beginn genau eine Münze. Es gibt zwei Typen von erlaubten Operationen:

Typ 1: Man wähle einen nicht-leeren Behälter $B_{j}$ mit $1 \leq j \leq 5$ aus. Man entferne eine Münze aus $B_{j}$ und füge zum Behälter $B_{j+1}$ zwei Münzen hinzu.

Typ 2: Man wähle einen nicht-leeren Behälter $B_{k}$ mit $1 \leq k \leq 4$ aus. Man entferne eine Münze aus $B_{k}$ und vertausche die Inhalte der (möglicherweise leeren) Behälter $B_{k+1}$ und $B_{k+2}$.

Man entscheide, ob es eine endliche Folge von solchen Operationen gibt, nach deren Ausführung die ersten fünf Behälter $B_{1}, B_{2}, B_{3}, B_{4}$ und $B_{5}$ leer sind und der sechste Behälter $B_{6}$ genau $2010^{2010^{2010}}$ Münzen enthält. (Man beachte: $a^{b^{c}}=a^{\left(b^{c}\right)}$.)

(Niederlande)

6. Es sei $a_{1}, a_{2}, a_{3}, \ldots$ eine Folge positiver reeller Zahlen. Ferner sei $s$ eine positive ganze Zahl, so dass

$$
a_{n}=\max \left\{a_{k}+a_{n-k} \mid 1 \leq k \leq n-1\right\}
$$

für alle $n>s$ gilt. Man beweise, dass es positive ganze Zahlen $N$ und $\ell$ mit $\ell \leq s$ derart gibt, dass $a_{n}=a_{\ell}+a_{n-\ell}$ für alle $n \geq N$ gilt.

(Iran)

\section{mathemas ordinate www.ordinate.de}

× 0431 23745-00/凶-01, info@ordinate.de $\rightarrow$ Software for mathematical people! Mathematica, ExtendSim, MathType, KaleidaGraph, Fortran, NSBasic, @ Risk und a.m.
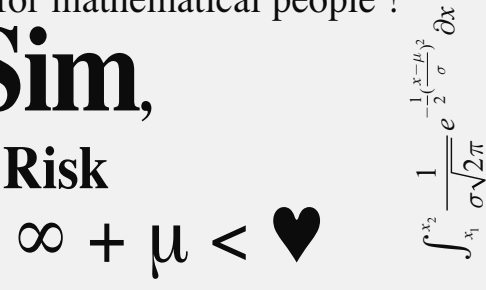

mathemas ordinate, Dipl. Math.Carsten Herrmann, M. Sc.

Königsbergerstr. 97, 24161 Altenholz

Mehr als 20 Jahre Erfahrung mit Software-Distribution! 